\title{
On the Implementation of the Integral Method for Residual Stress Measurement by Integrated Digital Image Correlation
}

\author{
A. Baldi \\ Dipartimento di Ingegneria Meccanica, Chimica e dei Materiali \\ Università degli Studi di Cagliari \\ Via Marengo, 2, I-09123 Cagliari, Italy \\ e-mail:antonio.baldi@dimcm.unica.it
}

\begin{abstract}
The Integrated Digital Image Correlation method (iDIC) is a simple and effective approach for residual stress measurement. iDIC differs from Digital Image Correlation because it replaces the "generic" displacement functions used to describe the displacement field around the measurement point with problemspecific ones. By this simple modification, stress components become the unknowns of the problem, thus allowing a single-pass analysis. Advantages are significant in terms of accuracy, robustness and ease of implementation. However, the implementation of the Integral Method for estimation of depth-dependent residual stress components is difficult.

This work suggests two alternative approaches to solve this problem; in the former, the direct solution of the triangular linear system is employed to
\end{abstract}


incrementally identify the stress distribution. In the latter, a global spatiotemporal minimization involving all the acquired images is suggested.

Keywords Integrated Digital Image Correlation, Residual Stress, Integral Method, Reverse Methods

\section{Introduction}

Most technological treatment induce in mechanical components a self balanced stress field, known as residual stress, as a side effect of the process.

Recognizing the presence of residual stress inside a component is particularly important because the field exists regardless of a load being applied to the components. Thus, it adds to load-induced stress fields, potentially inducing failure at load levels significantly lower than expected.

Various approaches to residual stress measurement have been proposed. The most used is the hole drilling technique [1, a semi-destructive technique consisting in drilling a small hole in the surface, to successively compute stress components from the strain/displacement field observed on the surface.

The above-cited technical norm assumes that the strains field is sampled using a strain gauge rosette, but various alternative measurement techniques, mostly optical, have been proposed. Even though in the past years both holographic interferometry [2,4] and grating interferometry [5] have been used to measure the displacement field around the hole, speckle interferometry is the most used interferometric technique in the present days [8 13]. However, all the interferometric techniques are highly sensitive to vibrations, thus making difficult their use in an industrial environment. A potential alternative is the Digital Image Correlation technique (DIC), a non-interferometric experimental technique. DIC is generally more tolerant environmentally than its interferometric counterparts, but it is also much less sensitive. Moreover, taking into 
account that the expected displacements for metallic materials are one pixel or less, it requires large subsets and a specific control logic [14] to limit the influence of outliers.

Integrated Digital Image Correlation (iDIC) is a non-interferometric optical technique [15, 16] which has been recently proposed as an effective approach for residual stress analysis both for isotropic [17] and orthotropic [18, 19] materials. Differently from standard DIC approach, iDIC makes use of a single subset; this is a significant advantage because the drilling process easily damages the painted speckles (due to chips, oil drops, fingerprints, ... ) thus, the analysis of a small region of the image could result in completely wrong data (which have to be detected and removed). This problem does not exist in iDIC, because a small erroneous region does not significantly affect the computation.

The iDIC technique is a direct derivative of Digital Image Correlation [20] and uses the same basic principle: given a pair of images acquired before and after the event of interest, the intensity of each pixel (respectively $f_{k}$ and $g_{k}$, where $k$ is the index of the pixel) remains the same, whatever the motion of the object under study. From the theoretical point of view, this statement can be written as $f\left(x_{k}, y_{k}\right)=g\left(x_{k}+u_{k}, y_{k}+v_{k}\right)$, where the displacement components $u$ and $v$ provide a simple approximation of the local behavior in DIC, whereas they are selected in such a way to be able to describe globally the displacement field related to the problem under analysis in iDIC.

The displacement mapping functions (e.g. $u=p_{0}+p_{1} \xi+p_{2} \eta+\ldots, v=$ $q_{0}+q_{1} \xi+q_{2} \eta+\ldots$ in standard DIC, where $\xi=x-x_{0}, \eta=y-y_{0}$ and $\left(x_{0}, y_{0}\right)$ is the origin of the local reference system) are controlled by a set of parameters (the $p_{l}$ and $q_{l}$ of the previous example) which are usually computed by minimizing over the area of the current subset a suitable error function [21], 
e.g. the Parametric Sum of Squared Difference (PSSD)

$$
\chi_{P S S D}^{2}=\sum_{k}\left(a+b f_{k}-g_{k}\right)^{2}
$$

where the coefficients $a$ and $b$ appearing in the $\chi_{P S S D}^{2}$ error criterion account for the offset and the scale change of the target system intensity and the summation ranges over all the pixels of the area under inspection.

To obtain a solution - the set of $p_{l}$ and $q_{l}$ minimizing the error functional - a Newton-Raphson approach is usually adopted: a linear approximation of either $f(x, y)$ or $g(x, y, u, v)$ is computed by expanding the intensity function in Taylor's series truncated to the first order, then a solution system is computed by setting to zero the derivatives of the error function with respect to the control parameters $p_{l}, q_{l}, a$ and $b$. The solution so obtained is not the correct one, because the coefficients of the linear system have been computed in the wrong location, thus, the algorithm is iterated and the sought solution is obtained by cumulating a series of (progressively smaller) corrections.

It is worth noting that the solution algorithm sketched above implies that a unique intensity pattern exists for each location, thus the surface of the specimen has to be textured either naturally or artificially (by spraying random speckles on the surface); moreover, the algorithm requires comparison of the value of the intensity at location $g\left(x_{k}+u_{k}, y_{k}+v_{k}\right)$ with $f\left(x_{k}, y_{k}\right)$ for each step of the iteration and for all points $k$ belonging to the current subset; thus, considering that both $u$, and $v$ are not ensured to be integers, $n$ interpolations are required for each step, where $n$ is the number of pixels belonging to the area under analysis (respectively the subset for the DIC technique and the active area of the image in the iDIC case).

The displacement field around a hole related to residual stress is well known: 


$$
\begin{gathered}
u_{r}=A\left(\sigma_{x}+\sigma_{y}\right)+B\left[\left(\sigma_{x}-\sigma_{y}\right) \cos (2 \theta)+2 \tau_{x y} \sin (2 \theta)\right] \\
u_{\theta}=C\left[\left(\sigma_{x}-\sigma_{y}\right) \sin (2 \theta)-2 \tau_{x y} \cos (2 \theta)\right] \\
u_{z}=F\left(\sigma_{x}+\sigma_{y}\right)+G\left[\left(\sigma_{x}-\sigma_{y}\right) \cos (2 \theta)+2 \tau_{x y} \sin (2 \theta)\right]
\end{gathered}
$$

where the $A, B, C, F$ and $G$ coefficients depend on material and location and are theoretically known ${ }^{1}$ in the hole through case [3], whereas they must be estimated using Finite Element computations in the blind hole case [11].

By projecting the in-plane displacement components $2 \mathrm{a}$ and $2 \mathrm{~b}$ ) in the $x-y$ reference system and taking into account the rigid body motion components $u_{0}$ and $v_{0}$, the residual-stress-specific iDIC shape functions can be computed:

$$
\begin{aligned}
& u\left(x, y, \sigma_{x}, \sigma_{y}, \tau_{x y}\right)=u_{0}+P^{u}(x, y) \sigma_{x}+Q^{u}(x, y) \sigma_{y}+T^{u}(x, y) \tau_{x y} \\
& v\left(x, y, \sigma_{x}, \sigma_{y}, \tau_{x y}\right)=v_{0}+P^{v}(x, y) \sigma_{x}+Q^{v}(x, y) \sigma_{y}+T^{v}(x, y) \tau_{x y}
\end{aligned}
$$

where $P^{u}, Q^{u}$ and $T^{u}\left(P^{v}, Q^{v}\right.$ and $\left.T^{v}\right)$ are calibration coefficients depending on point location, material properties and hole geometry:

$$
\begin{aligned}
& P^{u}=[A+(B+C) \cos (2 \theta)-C] \cos (\theta) \\
& Q^{u}=[A-B \cos (2 \theta)] \cos (\theta)+C \sin (2 \theta) \sin (\theta) \\
& T^{u}=+2[(B+C) \cos (2 \theta)+B] \sin (\theta) \\
& P^{v}=[A+(B+C) \cos (2 \theta)+C] \sin (\theta) \\
& Q^{v}=[A-(B+C) \cos (2 \theta)-C] \sin (\theta) \\
& T^{v}=-2[(B+C) \cos (2 \theta)-B] \cos (\theta)
\end{aligned}
$$

Once the iDIC shape functions (3) are known, the solution path exactly

\footnotetext{
${ }^{1}$ Equation 22 assumes a plane stress state and an linear elastic behavior of the material.
} 
follows the standard Lucas and Kanade formulation 22]; the solution matrix A and the known-terms vector $\mathbf{b}$ of the normal equation $\mathbf{A x}=\mathbf{b}$ can be estimated respectively as $\mathbf{A}=\sum_{k} \mathbf{h}_{k} \mathbf{h}_{k}^{\mathrm{T}}$ and $\mathbf{b}=-\sum_{k} g_{k} \mathbf{h}_{k}$, with

$$
\mathbf{h}_{k}=\left\{\begin{array}{c}
-1 \\
-f_{k} \\
\frac{\partial g_{k}}{\partial \xi} \\
\frac{\partial g_{k}}{\partial \eta} \\
\left.P_{u}\right|_{k} \frac{\partial g_{k}}{\partial \xi}+\left.P_{v}\right|_{k} \frac{\partial g_{k}}{\partial \eta} \\
\left.Q_{u}\right|_{k} \frac{\partial g_{k}}{\partial \xi}+\left.Q_{v}\right|_{k} \frac{\partial g_{k}}{\partial \eta} \\
\left.R_{u}\right|_{k} \frac{\partial g_{k}}{\partial \xi}+\left.R_{v}\right|_{k} \frac{\partial g_{k}}{\partial \eta}
\end{array}\right\}
$$

where the first and second row of (5) are related to the $a$ and $b$ coefficient of the $\chi_{P S S D}^{2}$ error function (1), the third and the fourth rows account for the rigid body motion components $\left(u_{0}\right.$ and $\left.v_{0}\right)$ and the last three are related to residual stress components $\left(\sigma_{x}, \sigma_{y}\right.$ and $\left.\tau_{x y}\right)$.

The solution matrix of iDIC is tiny $(7 \times 7$ when using the above described parameters, $8 \times 8$ if the in-plane rigid body rotation $\omega$ is included in the formulation [17) but the computation of each term of the matrix involves all the active pixels of the image. Thus, the iDIC approach to residual stress measurement is robust against noise [23, 24]. Moreover, it directly estimates the stress components, whereas almost all the other solution algorithms (both strain gauge and optical based) perform a two-step procedure: first the displacements (strains) are measured in three (or more) points, then the residual stress components are estimated by solving an inverse problem. However, the single-pass solution becomes a problem when residual stress components depend on depth.

The standard approach to the identification of a residual stress field varying with depth is the integral method suggested by Schajer [25] in 1988. The de- 

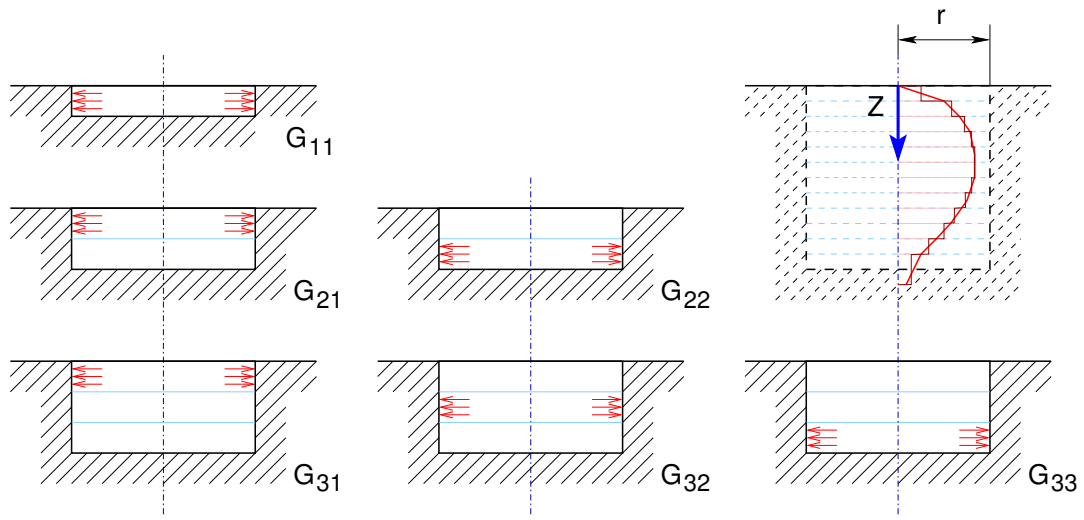

Figure 1: The Integral Method: schematic representation of the computation steps.

pendence on depth is approximated with a stepwise distribution (Figure 1). For each step, a hole increment is performed and the strain/displacement field on the surface is acquired. To correlate the observed displacements with residual stress, one has to consider that the displacement/strain field depends on the newly released stress components and on all the previously released ones. This leads to the linear system

$$
\mathbf{G} \sigma=\mathbf{f}
$$

where $\boldsymbol{\sigma}$ is the array of stress components, $\mathbf{f}$ is the vector of the strains / displacements measured on the surface and $\mathbf{G}$ is the influence matrix. The entry $G_{i, j}$ of $\mathbf{G}$ conceptually is the component of deformation/displacement observed on the surface after $i$ hole increments due to a unitary stress at depth $j$. As no influence is assumed on stress below the bottom of the hole, $\mathbf{G}$ is lower triangular (i.e. $G_{i, j}=0$ if $j>i$ ). Moreover, as a plane stress status is described by three tensor components, at least three points of the surface have to be sampled, thus, $G_{i, j}$ actually is a sub-matrix (each row corresponding to a different sampled point ${ }^{2}$ ).

\footnotetext{
${ }^{2}$ Depending on the formulation, the matrix $G_{i, j}$ may be diagonal, thus allowing for solution of three independent linear systems.
} 
It is worth noting that even though $\mathbf{G}$ is usually square (a residual stress rosette contains three active elements), it is not always so: when using optical methods, each of the $n$ pixels of the acquired image potentially provides an independent measurement, thus $G_{i, j}$ may have up to $n$ rows and consequently, sizeof $(\mathbf{f})=n \cdot m$, where $m$ is the number of material removals (i.e. hole depth increments).

The overdetermined linear system $-\mathbf{G}$ results in $(n \cdot m)$ rows by $(3 \cdot m)$ columns, $n \gg m$ - can be solved by Singular Value Decomposition (SVD) or by a Least Squares approach, i.e. by pre-multiplying both sides of equation (6) by $\mathrm{G}^{\mathrm{T}}$

$$
\mathbf{G}^{\mathrm{T}} \mathbf{G} \boldsymbol{\sigma}=\mathbf{G}^{\mathrm{T}} \mathbf{f}
$$

and solving the resulting $(3 \cdot m) \times(3 \cdot m)$ linear system by a standard method.

In principle, the integral method does not depend on the experimental approach, thus (6) should apply to the iDIC method. Actually, its use in combination with iDIC is difficult because displacements (strains) are never explicitly computed, thus, the vector $\mathbf{f}$ appearing in $(6)$ is not known. Indeed, in the iDIC formulation the known terms are the pixel intensities estimated in the displaced configuration. As displacements are a function of residual stress components (i.e. of the unknowns), the direct use of (6) is not possible: the integral approach has to be incorporated inside the solution algorithm.

This work focuses on the implementation of the above sketched idea and is organized as follows: the next section is devoted to the development of a spatiotemporal iDIC formulation to overcome the above noted limitation. Next, stability issues and algorithm variants are discussed (the integral method is well known for producing ill-conditioned matrix). In the same section the identification of an initial stress profile, to be used as alternative initial guess, is also discussed. The next section focuses on the validation of the proposed ap- 
proaches first by using synthetic images, then, by an experimental approach. The performance of the various algorithms is compared and some critical points are highlighted in the following section.

Appendix $\mathrm{A}$ discusses the numerical implementation of the algorithms, focusing on memory requirements and execution time.

\section{A Spatio-Temporal Approach to Residual Stress Measurement}

The solution algorithm of the integral method searches for the best stress profile, i.e. the stress distribution with the depth which minimizes the errors between the computed displacements and the experimental measurements across the full drilling history. Thus, the iDIC objective function (1) has to be extended in such a way to depend on all $m$ drilling increments at the same time:

$$
\chi_{i P S S D}^{2}=\sum_{i=1}^{m} \sum_{k=1}^{n}\left(a_{i}+b_{i} f_{k}-g_{i, k}\right)^{2}
$$

where

$$
g_{i, k}=g\left(x_{k}+u_{i, k}, y_{k}+v_{i, k}\right)
$$

and

$$
\begin{aligned}
& u_{i, k}=u_{i}+\sum_{j=1}^{i} P_{i, j, k}^{u} \sigma_{x, j}+Q_{i, j, k}^{u} \sigma_{y, j}+T_{i, j, k}^{u} \tau_{x y, j} \\
& v_{i, k}=v_{i}+\sum_{j=1}^{i} P_{i, j, k}^{v} \sigma_{x, j}+Q_{i, j, k}^{v} \sigma_{y, j}+T_{i, j, k}^{v} \tau_{x y, j}
\end{aligned}
$$

In the above equations, the $i$ index refers to the drilling increment (i.e. to the acquired image), the $j$ counter addresses the loading step (thus, $j \leq i$ ) and 
$k$ identifies a specific location of the image (a pixe ${ }^{3}$ ).

The $\chi_{i P S S D a b}^{2}$ function (8) estimates the (cumulated) discrepancy between the reference image and all the experimental images acquired during the material removal history. To this aim, every pixel $k$ corresponding to point $\left(x_{k}, y_{k}\right)$ in the reference image is located in each of the $m$ images acquired during the material removal procedure by adding a displacement vector (eq. 9) depending on all stress components released up to image index $i$ (i.e. all $\sigma_{x, j}, \sigma_{y, j}, \tau_{x y, j}, \forall j \leq$ i). Thus, the optimization algorithm tries to minimize the difference between what is observed experimentally and what should be acquired given the current residual stress profile and the theoretical displacement model hardcoded inside it (through the calibration coefficients). To obtain this objective, it adjusts all the residual stress values and all rigid body motion components simultaneously, thus performing both an optimization in space (the area around the hole) and in time (through the entire drilling history) [26, 27.

A few final notes about the proposed algorithm:

- The $u_{i}$ and $v_{i}$ appearing in $9 \mathrm{a}$ and $9 \mathrm{~b}$ are the rigid body motion components related to material removal step $i$ : indeed, depending on the experimental protocol, either the specimen or the camera may move between the drilling steps 4

- in the same way, the lighting condition of each image may change, thus we have to include a mean intensity and contrast coefficient $\left(a_{i}, b_{i}\right)$ for each drilling increment;

- the $P_{i, j, k}^{u}, Q_{i, j, k}^{u}, \ldots, T_{i, j, k}^{v}$ are the equivalent of the $G_{i, j}$ in the "standard"

\footnotetext{
${ }^{3} \mathrm{By}$ selecting either a row-major or column-major ordering it is possible to use a single index to uniquely identify a pixel of an image; to give an example, the pixel at row 3 and column 2 can be indexed either as $3 \times w+2$ (row-major) or $2 \times h+3$ (column-major), where $w$ and $h$ respectively are the width and the height of the image in pixels.

${ }^{4}$ In principle, adding linear terms to $u$ and $v$ may help correcting micro-rotations of the camera. Actually this should be avoided because the residual stress displacement field is an odd function, thus, as a close view of the area around the hole is usually acquired, the fitted plane will never be horizontal, even when no correction is required.
} 
integral method and depend, apart from material parameters, on the location $(k)$, the stress layer $(j)$ and the current geometry (i.e. drilling step $i$ ); due to the blind hole configuration, they have to be computed by Finite Element simulations, obviously using the same material removal sequence of the current experiment;

- even though the construction of the solution matrix involves a large data set (all the images acquired during the drilling process), the matrix itself is not large: each drilling increment requires 7 degrees of freedom, thus, the total matrix size is $(7 \cdot m) \times(7 \cdot m)$.

\section{Algorithm Implementation and Stability Is- sues}

The integral method is known to be highly sensitive to noise; in mathematical terms, the solution matrix is ill-conditioned. This is partially due to the solution algorithm (the least-squares method) but is mostly related to a physical problem: the deeper the released stress, the smaller the displacements observed on the surface, thus making a reliable measurement progressively harder.

By trying to reconstruct a variable stress profile, iDIC has to face the same physical limitation and, in the same way as the integral method, its solution matrix becomes ill-conditioned, thus making the solution of (8) unstable in the presence of noise. The next subsections discusses algorithm initialization, solution regularization and alternative solution algorithms aiming to improve the stability of the algorithm. 


\subsection{Stress-Profile Initialization}

The above-described algorithm shares with "standard" iDIC the ability to converge to the expected solution starting from a null stress field 5 Even though this behavior turns out to be useful, considering the iterative (i.e. nonlinear) nature of the algorithm, one may wonder if a better final solution can be obtained starting from a different initial distribution of the stress components.

Actually, considering that the matrix $\mathbf{G}$ is lower triangular, an initial estimate can be easily computed. In fact, equations $9 \mathrm{a}$ and $9 \mathrm{~b}$, expressing the displacement $(u, v)$ expected at point $k$ of the surface at drilling increment $i$, can be rearranged as

$$
\begin{aligned}
u_{i, k}=u_{i} & +\left[\sum_{j=1}^{i-1} P_{i, j, k}^{u} \sigma_{x, j}+Q_{i, j, k}^{u} \sigma_{y, j}+T_{i, j, k}^{u} \tau_{x y, j}\right] \\
+ & P_{i, i, k}^{u} \sigma_{x, i}+Q_{i, i, k}^{u} \sigma_{y, i}+T_{i, i, k}^{u} \tau_{x y, i} \\
v_{i, k}=v_{i}+ & {\left[\sum_{j=1}^{i-1} P_{i, j, k}^{v} \sigma_{x, j}+Q_{i, j, k}^{v} \sigma_{y, j}+T_{i, j, k}^{v} \tau_{x y, j}\right] } \\
+ & P_{i, i, k}^{v} \sigma_{x, i}+Q_{i, i, k}^{v} \sigma_{y, i}+T_{i, i, k}^{v} \tau_{x y, i}
\end{aligned}
$$

where we have singled out the last drilling increment.

If you assume to know the stress components for all drilling increments up to the previous one (i.e. up to $i-1$ ), equation 10 clearly shows that their contribution to the current displacement is completely known, because it is estimated by the terms in brackets (the summation), which depend on known stress components and on the calibration coefficients related to the current geometric configuration only.

\footnotetext{
${ }^{5}$ Note that displacements related to residual stress asymptotically tend to zero with distance from the center of the hole; thus, providing the rigid body motion is correctly estimated, the area far from the hole is almost correct even when the contribution from residual stress is not included.
} 
Thus, equation 10 actually reads as

$$
\begin{aligned}
& u_{i, k}=u_{i}+u_{i, k}^{p}+P_{i, i, k}^{u} \sigma_{x, i}+Q_{i, i, k}^{u} \sigma_{y, i}+T_{i, i, k}^{u} \tau_{x y, i} \\
& v_{i, k}=v_{i}+v_{i, k}^{p}+P_{i, i, k}^{v} \sigma_{x, i}+Q_{i, i, k}^{v} \sigma_{y, i}+T_{i, i, k}^{v} \tau_{x y, i}
\end{aligned}
$$

where $u_{i, k}^{p}$ and $v_{i, k}^{p}$ are the displacements due to the (known) previously released residual stresses.

The algorithm to estimate an initial residual stress distribution is straightforward: at the beginning of the process $u_{1, k}^{p}$ and $v_{1, k}^{p}$ are identically null, thus the stress components can be computed using a "standard" iDIC algorithm. At the second drilling increment, the residual stress components related to the first step are known, thus the summations in the brackets can be evaluated and their contribution added to the displacement field; the remaining quantities only depend on stress components related to the second material layer, which can be easily computed using the $u_{i, k^{-}}^{p} v_{i, k^{-}}^{p}$ augmented version of the iDIC algorithm 6 . The solution process continues in the same way step-by-step; the full process consisting of $m$ executions of a "standard" iDIC algorithm intermixed with $m-1$ computations of the $u_{i}^{p}$ and $v_{i}^{p}$ values at point $k$.

It is worth noting that the "initialization" algorithm described in this section actually results in a complete description of the stress profile, thus, it can be viewed as an alternative way of measurement (named the fast algorithm from now on). It compares favorably with the previously described algorithm (full algorithm) in terms of memory storage and computation requirements, but presents two main drawbacks: its sequential approach makes it impossible to use Tikhonov regularization (described in the following) and it propagate errors: indeed, a misidentified stress level affect all the following steps. Indeed, to compensate for an erroneous stress estimate at step $i$, the algorithm will

\footnotetext{
${ }^{6}$ The only modification is the inclusion of $u_{i, k}^{p}$ and $v_{i, k}^{p}$ in the evaluation of the coordinate in the target image.
} 
overcompensate the stress evaluation at step $i+1$, thus inducing an oscillatory behavior around the correct solution.

\subsection{Use of the Singular Value Decomposition}

SVD can be used in two different ways to help solve the ill-conditioning problem: it can be employed either as a "recovery" tool, i.e. as an alternative way to solve the normal equation, or it can be used to directly identify the best-fit solution without assembling the matrix A. The former path requires assembling the normal equation matrix in the standard way and then decomposing it via SVD, i.e. writing $\mathbf{A}=\mathbf{U} \mathbf{W} \mathbf{V}^{\mathrm{T}}$, where $\mathbf{U}$ is a column-ortogonal matrix, $\mathbf{W}$ is a diagonal matrix containing the (positive or null) singular values $w_{j}$ and $\mathbf{V}$ is an orthogonal matrix. It can be shown that the decomposition is always possible, thus, the inverse of $\mathbf{A}$ can always be computed as

$$
\boldsymbol{A}^{-1}=\mathbf{V}\left[\operatorname{diag}\left(1 / w_{j}\right)\right] \mathbf{U}^{\mathrm{T}}
$$

Solving the normal equation via SVD leads to exactly the same results as using LU decomposition when $\mathbf{A}$ is well- or mildly ill-conditioned, but allows for removal of singular eigenvectors from the solution in critical situations. This can be easily performed by replacing $1 / w_{j}$ with 0 when the ratio of $w_{j}$ to $\max \left(w_{j}\right)$ is below a given threshold 7 .

Note that the SVD decomposition of an $n_{r} \times n_{c}$ matrix requires $c_{1} n_{r}^{2} n_{c}+c_{2} n_{c}^{3}$ operations, where $c_{1}=4$ and $c_{2}=22$ for the R-SVD algorithm [29]. This is significantly more than the $2 / 3 n_{r}^{3}$ required by LU; nevertheless, $n_{r}$ is so small $\left(n_{r}=n_{c}=7 m\right.$ in the normal-equation case) that the SVD decomposition requires negligible time.

\footnotetext{
${ }^{7}$ Selection of the threshold $t$ is a critical point; in this work we assumed $t=$ $(1 / 2) \max \left(w_{j}\right) \varepsilon \sqrt{n_{r}+n_{c}+1}$, where $\varepsilon$ is the expected roundoff error [28].
} 
The least-squares problem can also be directly solved by SVD. It suffices to decompose via SVD and successively invert the $(n \cdot m) \times(7 \cdot m)$ matrix resulting from writing the $n \cdot m$ equations corresponding to each value of the $i$ and $k$ indexes in (8) (obviously without squaring). Indeed, 28] shows that in the case of an overdetermined system, SVD produces a solution that is the best approximation in the least-squares sense. In principle, this is the most suitable approach to the solution of least-squares problems, because computing the normal equation squares the conditions number, however, in the iDIC case it results to be highly numerically intensive because the solution matrix can easily have a million (or more) rows ( $n$, the number of active pixels of each image, is $\mathcal{O}\left(10^{5}\right)$ and (8) involves all the images at the same time); moreover the iDIC algorithm is iterative, thus, several solution steps may be required.

\subsection{Tikhonov Regularization}

Tikhonov regularization [30] enforces a smoother solution by applying a penalty function to the solution. To this end, the solution matrix is augmented with an extra term which penalizes a chosen derivative of the solution.

Using this approach, equation 7 becomes

$$
\left(\mathbf{G}^{\mathrm{T}} \mathbf{G}+\lambda \mathbf{c}^{\mathrm{T}} \mathbf{c}\right) \boldsymbol{\sigma}=\mathbf{G}^{\mathrm{T}} \mathbf{f}
$$

where $\lambda$ is the penalty coefficient and the matrix $\mathbf{c}$ implements (in discrete form) the penalized function (in this case the curvature of the solution, i.e. the second 
derivative).

$$
\mathbf{c}=\left[\begin{array}{cccccc}
0 & 0 & 0 & 0 & \ldots & 0 \\
-1 & 2 & -1 & 0 & \ldots & 0 \\
0 & -1 & 2 & -1 & \ldots & 0 \\
\vdots & & & \ddots & & \\
0 & 0 & \ldots & -1 & 2 & -1 \\
0 & 0 & \ldots & 0 & 0 & 0
\end{array}\right]
$$

Our implementation strictly follows [31, 32] with the obvious modification that $\mathbf{c}$ acts only on the rows of the matrix related to stress components (i.e. all the rows of matrix $\mathbf{c}$ present the $-1,2,-1$ sequence on the diagonal, except for the first, the last and those related to the intensity parameters or to the rigid body motion components which are identically null).

We implemented Tikhonov regularization in the context of the normal equation only. In principle the resulting matrix can be solved using SVD, but the introduction of the penalizing term significantly improves the condition number, thus making the use of SVD valueless.

\subsection{Incremental Approach}

Schajer and Rickert 33 have shown that a better condition number of matrix $\mathbf{G}$ (equation 7) can be obtained by using an incremental approach. The basic idea consists in "using incremental deformation data instead of the total deformation data that are conventionally used." In the same way as in the previously described approaches, a direct mapping of this formulation to iDIC is not possible; however, a spatio-temporal minimization based on this idea can be formulated. To this aim, it suffices to replace the reference image $f$ appearing in the $\chi^{2}$ error 
criterion (eq. 8) with the previously acquired image $g_{i-1}$ :

$$
\chi^{2}=\sum_{i=1}^{m} \sum_{k=1}^{n}\left(a_{i}+b_{i} g_{(i-1), k}-g_{i, k}\right)^{2}
$$

and replace the $P_{i, j, k}^{u}, \ldots, T_{i, j, k}^{v}$ appearing in 9 with their incremental counterparts $\tilde{P}_{i, j, k}^{u}, \ldots, \tilde{T}_{i, j, k}^{v}$, i.e 8 ,

$$
\begin{aligned}
& u_{i, k}=u_{i}+\sum_{j=1}^{i} \tilde{P}_{i, j, k}^{u} \sigma_{x, j}+\tilde{Q}_{i, j, k}^{u} \sigma_{y, j}+\tilde{T}_{i, j, k}^{u} \tau_{x y, j} \\
& v_{i, k}=v_{i}+\sum_{j=1}^{i} \tilde{P}_{i, j, k}^{v} \sigma_{x, j}+\tilde{Q}_{i, j, k}^{v} \sigma_{y, j}+\tilde{T}_{i, j, k}^{v} \tau_{x y, j}
\end{aligned}
$$

It is worth noting that the same modifications can be performed to the fast formulation described in sec. 3.1 (i.e. replace the $P^{u} \ldots T^{v}$ coefficients by $\tilde{P}^{u} \ldots \tilde{T}^{v}$ and use $g_{i-1}$ as reference image) thus providing a new solution algorithm. Note that both the SVD solutions and the Tikhonov regularization can be used in combination with 12 .

\section{Numerical Validation}

In the previous sections we described the basic formulation and various modifications to the basic idea. The number of variants is significant because some of the proposed "improvements" can be used at the same time. Thus, before performing a real experiment, we opted for using numerically synthesized images because in this way the expected results are completely known and the accuracy of the various algorithms or algorithm combinations can be evaluated. However, image synthesizing requires some care because we have to avoid interpolation (it is well known that this procedure biases the data) and we cannot refer to

\footnotetext{
${ }^{8}$ The incremental coefficients $\tilde{P}_{i, j, k}^{u}, \ldots, \tilde{T}_{i, j, k}^{v}$ can be computed starting from the absolute ones by subtracting from each element at row $i$ the corresponding value at row $i-1$.
} 
Table 1: Assumed stress profile (and rigid body motion)

\begin{tabular}{c|ccl|cc}
\hline $\begin{array}{c}\text { Depth } \\
\mathrm{mm}\end{array}$ & $\begin{array}{c}\sigma_{x} \\
\mathrm{MPa}\end{array}$ & $\begin{array}{c}\sigma_{y} \\
\mathrm{MPa}\end{array}$ & $\begin{array}{c}\tau_{x y} \\
\mathrm{MPa}\end{array}$ & $\begin{array}{c}u_{0} \\
\text { pixel }\end{array}$ & $\begin{array}{c}v_{0} \\
\text { pixel }\end{array}$ \\
\hline 0.05 & 180 & 120 & 100. & 0.0 & 0.0 \\
0.10 & 195 & 125 & 105. & 10.1 & 10.35 \\
0.15 & 200 & 129 & 110. & 9.7 & 8.2 \\
0.20 & 190 & 132 & 115. & -10.44 & 8.3 \\
0.25 & 175 & 134 & 120. & 5.55 & -3.02 \\
0.30 & 160 & 135 & 124.5 & 15.3 & 10.76 \\
0.35 & 150 & 134 & 129. & -7.1 & -8.32 \\
0.40 & 140 & 132 & 132.5 & -12.56 & -15.28 \\
0.45 & 132 & 129 & 135. & 18.11 & 13.3 \\
0.50 & 126 & 125 & 136.5 & -15.543 & 13.24 \\
0.55 & 122 & 120 & 137. & 10.02 & -12.76 \\
0.60 & 120 & 115 & 137. & 5.98 & 5.9 \\
0.65 & 118 & 109 & 137. & -5.4 & 5.22 \\
\hline
\end{tabular}

the hole-drilling theoretical solution (it assumes a through-hole configuration).

To solve this problem we assumed that each speckle of the reference picture can be described as a bell-shaped function [34 37]:

$$
b(r)= \begin{cases}s\left[1-(r / \zeta)^{2}\right]^{3} & r \leq \zeta \\ 0 & \text { elsewhere }\end{cases}
$$

where $s$ is the scale factor and $\zeta$ is the radius of the speckle. Consequently, the speckle field can be viewed as the superposition of several translated and scaled bell functions, i.e. the resulting reference intensity field is continuous? Thus, it is possible to use whatever sampling rate when generating the displaced images; this allows for satisfaction of the requirements of the Nyquist-Shannon sampling theorem whatever the displacement field 10

The displacement field is still described by (9), but both the stress profile

\footnotetext{
${ }^{9}$ The parameters of the speckles are generated from user-defined statistical distributions and stored for later use.

${ }^{10}$ Note that by using a large enough oversampling it is possible to avoid identification of the reverse mapping function, thus, making image generation simpler.
} 


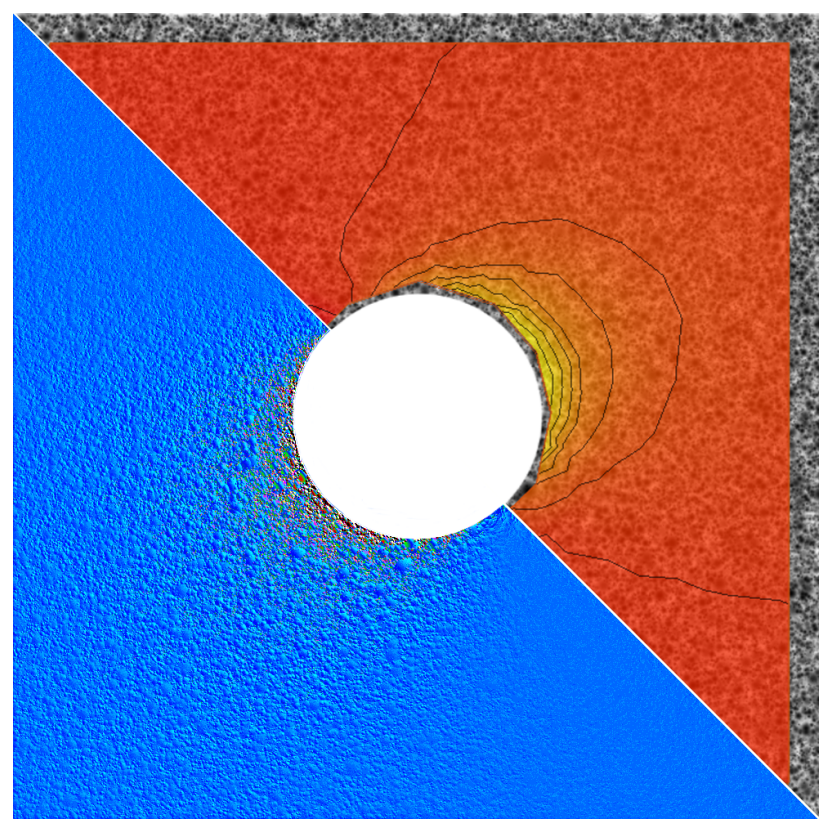

Figure 2: An example of synthetic image. Top right part: $u$ displacement field of the first drilling increment computed by a standard DIC code. Bottom left: subtraction of first image and the reference one (note that a false-color palette was employed to simplify visualization). Displacements can be recognized by this technique because their maximum value is about one pixel. Nevertheless, noise masks almost all the signal apart from the area near the hole.

and the rigid body displacements have to be completely known. Moreover, the calibration coefficients $P_{i, j, k}^{u}, \ldots, T_{i, j, k}^{v}$ have to comply with the material removal sequence at each increment both in terms of depth and geometrical parameters.

Table 1 reports the prescribed stress components. During generation, a $3 \mathrm{~mm}$ hole diameter and a constant drilling increment $(0.05 \mathrm{~mm})$ have been used. The image size is $1024 \times 1024 \mathrm{pixel}^{2}$, the intensity has been quantized using 10 bit and up to 4 bit have been perturbed using uniform distributed noise. Moreover, random rigid body motion components have been added for each drilling increment (see columns 5 and 6 of Table 1). The assumed pixel side was $10 \mu \mathrm{m}$, thus the imaged area corresponds to about $10 \times 10 \mathrm{~mm}^{2}$. 
Figure 2 shows, in the top right part, the $u$ displacement field computed by a "standard" DIC code 11 , and the subtraction of the first image and the reference one is shown in the bottom left area. To make recognizing the details easier, a false-color palette was used in the latter case. Note that, as the assumed Young's modulus is $70 \mathrm{GPa}$, maximum displacement is quite small (less than 1 pixel).

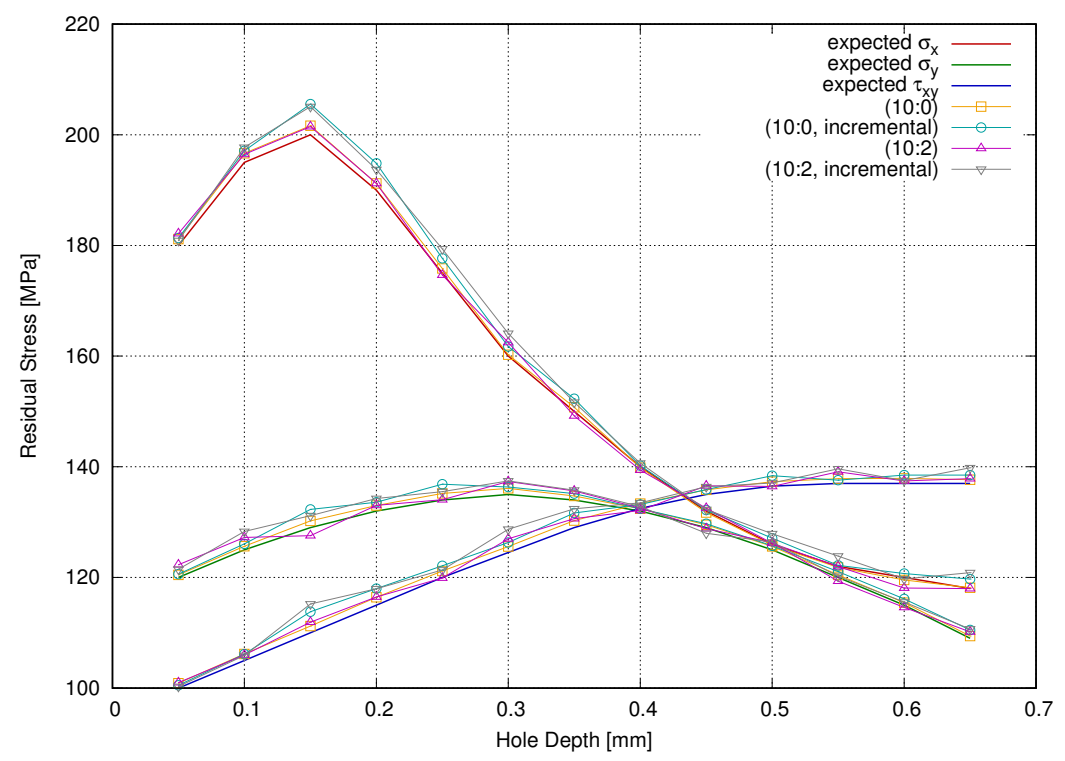

Figure 3: Synthetic images. Expected vs estimated results; integer rigid body motion. The $q$ in the $(q: n)$ code refers to the number of bits used for quantizing the intensity, whereas the number after the colon is the number of lower bits affected by noise.

Figures 3 shows results of first test, i.e. the expected and the measured residual stress estimated using the described algorithms. Given that all stress components behave the same, Figure 4 and following show the $\sigma_{x}$ stress component only, to make it simpler reading results. To simplify the analysis, integer rigid body motion displacement components have been assumed (i.e. the as-

\footnotetext{
${ }^{11}$ Analysis was performed using a global code employing triangular elements and an unstructured mesh.
} 


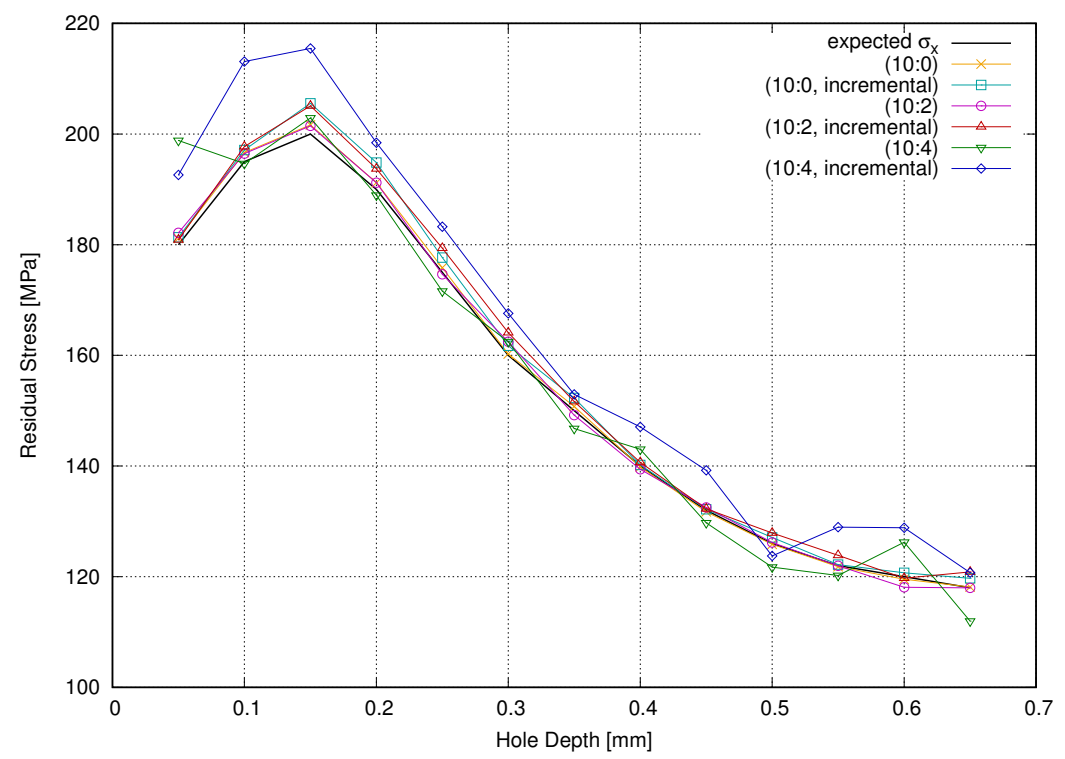

Figure 4: Synthetic images. Expected vs estimated results; magnified view of the $\sigma_{x}$ stress component.

sumed rigid body motion components are $\left\lfloor u_{0}\right\rfloor,\left\lfloor v_{0}\right\rfloor$ where $u_{0}$ and $v_{0}$ can be found in Table 11. Indeed, by this assumption the influence of the $u_{i}$ and $v_{i}$ terms appearing in $(9)$ is minimized.

Looking at Figure 4 it is apparent that all the proposed algorithms give the expected results when little or no noise is present ( 0 or 2 noise bits): results of the fast and full algorithms are practically identical (all estimated stress values differ for less than one $\mathrm{MPa}$ ) and the use of the SVD solver gives no advantage, because all singular values are significant. Results are quite smooth, thus Tikhonov stabilization is not required.

Quite interestingly, results of the incremental approach are worse than those of the absolute one when little or no noise is present. This appears to conflict with theory (using an incremental approach should give a better conditioned matrix). However it should be noted that incremental displacements are significantly smaller than absolute ones, thus, when no other error source is present, 
the uncertainty of DIC-estimated displacements becomes significant.

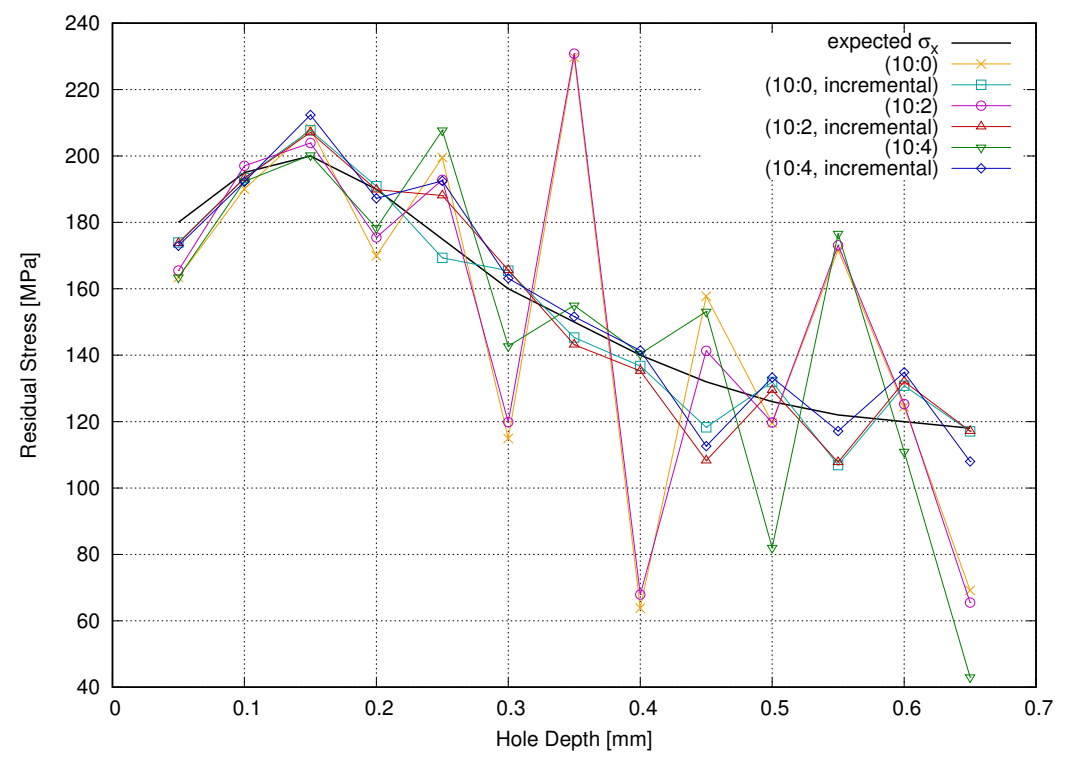

Figure 5: Synthetic images; expected vs estimated results. Fractional rigid body motion. As results are significantly noisier, only the $\sigma_{x}$ component is shown.

Figure 5 shows the estimated residual stress when the assumed rigid body motion components are fractional. As results are significantly noisier, only the $\sigma_{x}$ component is shown (results related to $\sigma_{y}$ and $\tau_{x y}$ are qualitatively the same); in the same way, Tikhonov-related results have been postponed to the next image.

Looking at Figure 5 the advantage of incremental formulation becomes apparent: results still are somewhat oscillating, but differently from absolute analysis, no significant spike appears. It is work noting that results of the fast and full algorithms are still mostly the same and that the use of the SVD solver (both variants) gives no advantage.

Figure 6 analyzes the influence of Tikhonov stabilization. In principle, $\lambda$ should be selected using the Morozov criterion [31] however, to show the influence of $\lambda$ on the solution, various analyses have been performed using $\lambda$ 

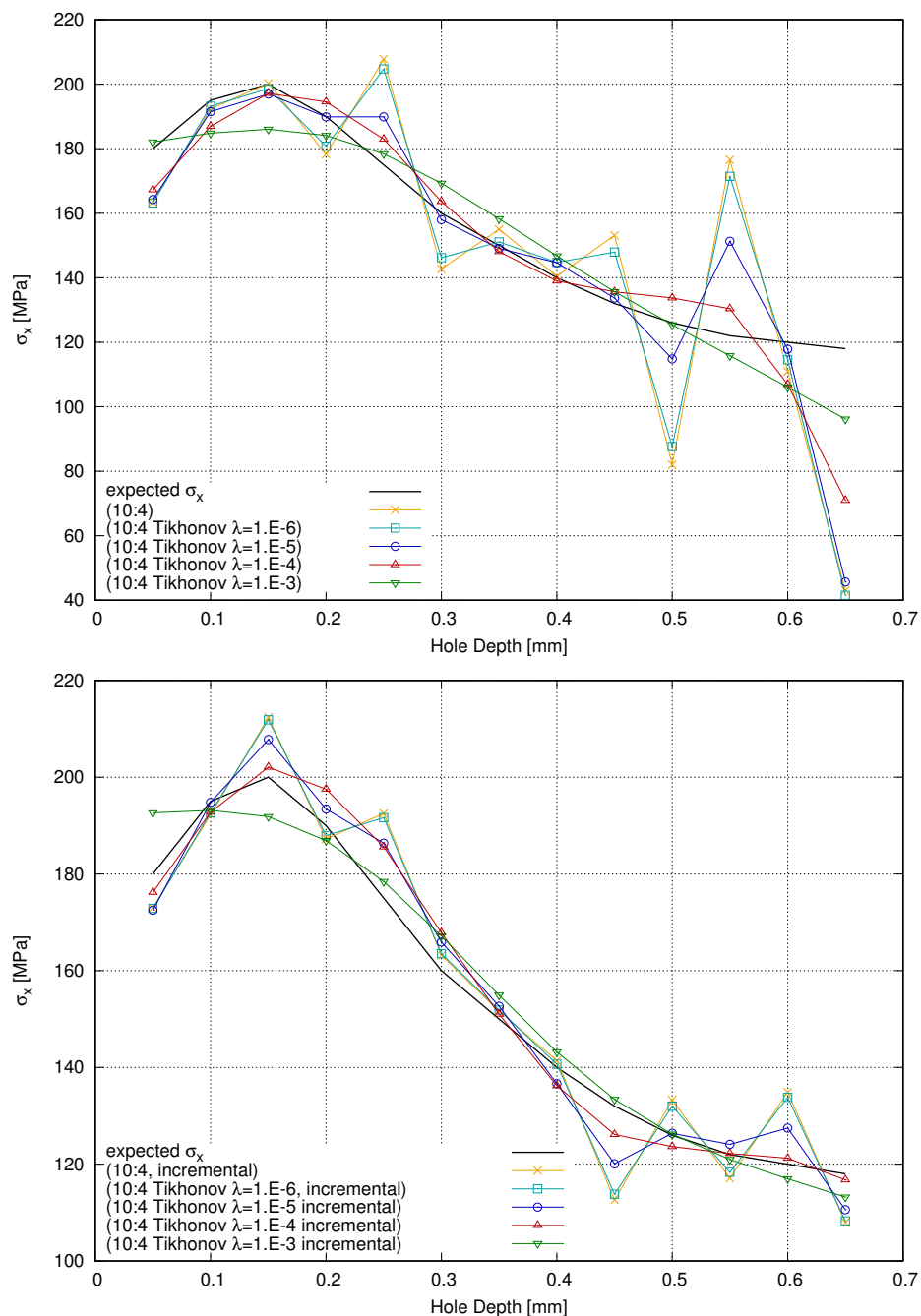

Figure 6: Synthetic images, expected vs estimated results; fractional rigid body motion. Top: influence of Tikhonov regularization on the absolute formulation. Bottom: same analysis, incremental formulation. Note that only the $\sigma_{x}$ stress component is shown.

values ranging from $10^{-6}$ up to $10^{-3}$. Figure 6 top shows results of the use of Tikhonov regularization in combination with the absolute formulation while Figure 6 bottom is related to the incremental algorithm. In both cases Tikhonov regularization allows smoothing results. In the same way as the "standard" in- 
tegral method, the higher the value of $\lambda$, the smoother and the more biased the results. It is worth noting that as the incremental algorithm is inherently more stable, the same smoothing level can be obtained using a significantly lower value of $\lambda$. Finally, it should be remembered that Tikhonov regularization cannot be applied to the fast algorithm due to the incremental computation.

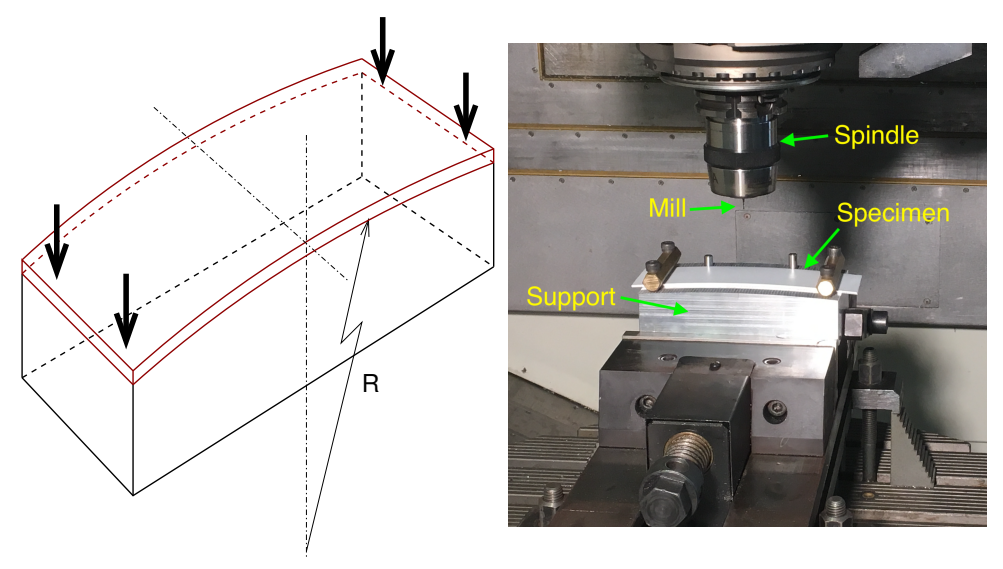

Figure 7: Left: sketch of the experimental setup. Right: actual setup.

\section{$5 \quad$ Experimental Validation}

To experimentally assess the proposed approach, we followed a procedure similar to 32]: two specimens were cut from a $2000 \times 1.85 \times 40 \mathrm{~mm}$ aluminum alloy plate $($ length $\times$ thickness $\times$ width $)$ and the proposed experimental protocol was applied on both, using a flat reference surface for the former and an assumed curvature support for the latter. To this end, a $70 \times 200 \times 40 \mathrm{~mm}$ aluminum block $($ width $\times$ length $\times \min ($ thickness $))$ was machined, with a constant curvature top surface (see Figure 7 left), thus imposing a linear stress profile inside the 
specimen. A numerically controlled milling machine ${ }^{12}$ was used to drill the specimens and the acquisition of the area around the hole before and after milling ${ }^{13}$ was performed using an Allied Vision Pike F421 camera installed on the milling head. Finally an artificial speckle field was painted on the surface of the specimens.
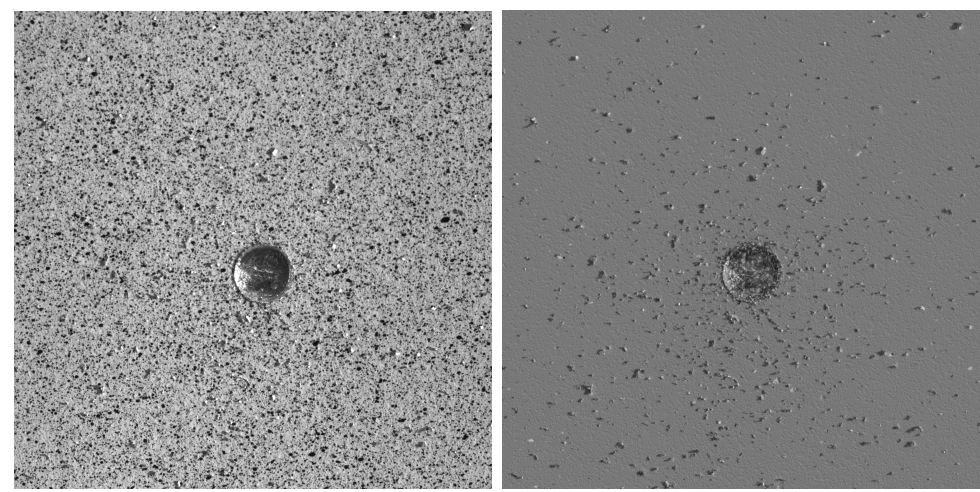

Figure 8: Residual metallic chips after a $0.05 \mathrm{~mm}$ drilling increment. Left: actual image; right: difference of the current image and the reference image.

Figure 8 left shows the surface of the specimen immediately after milling (i.e. before removing chips by an air jet). Even though it is not easy to detect them, a close look shows that several metallic micro-chips are present. Indeed, to identify them it suffices to subtract the reference image from Figure 8 -left, thus obtaining Figure 8 right. Even though it is easy to blow most of them out, nevertheless we opted for building the list of active pixels by computing the absolute difference of the normalized image intensity and the reference one. In more detail, for each pixel $k$ of each image $i$ we computed

$$
\Delta_{i, k}=\left|g_{i}\left(x_{k}+\left\lfloor u_{i}\right\rfloor, y_{k}+\left\lfloor v_{i}\right\rfloor\right)-f\left(x_{k}, y_{k}\right)\right|
$$

\footnotetext{
${ }^{12}$ Deckle Maho DMU 60 P hi-dyn.

${ }^{13}$ Milling was performed using a $1 \mathrm{~mm}$ diameter endmill (Sandvik-Coromant 1 P230-0100XA 1630) spinning at maximum speed allowed by the spindle (18000 RPM).
} 
As (13) accounts for integer rigid body motion components, $\Delta_{i, k}$ is very small unless a significant difference (a metallic chip, a grain of powder, a drop of lubricant fluid, a defect of painted layer) is present. Thus, we disabled all pixels $k$ whenever $\Delta_{i, k} \geq c$, where we assumed $c=25 \%$ during our analyses.

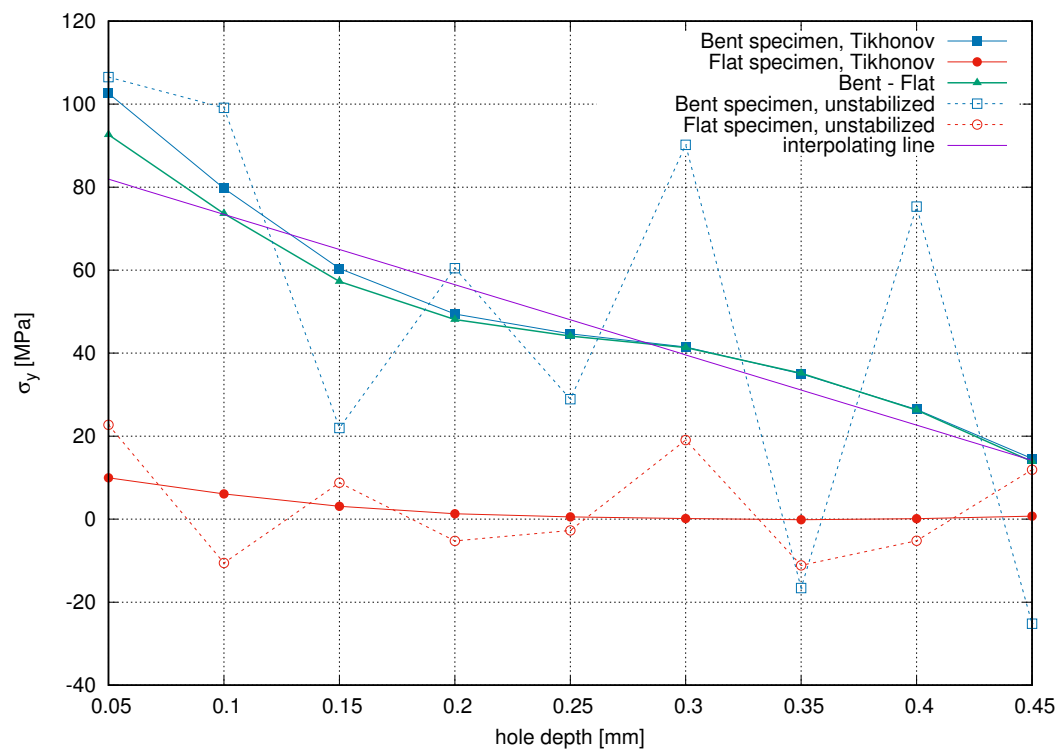

Figure 9: Experimental results; the dashed lines are estimated using the full algorithm with no modification, while the continuous lines with dots results from using Tikhonov regularization $\left(\lambda=10^{-3]}\right)$.

Figure 9 shows results of the experimental analysis for both the flat and the curved supports: the dashed lines correspond to the use of the plain vanilla full algorithm (i.e. using (8) with LU solver) and show significant oscillations ${ }^{14}$ Using Tikhonov regularization smooths results; with the assumed $\lambda=1 \cdot 10^{-3}$, only a small, long period, residual oscillation remains, whereas smaller $\lambda$ values are unable to stabilize the algorithm. Note that all the analyses were performed using the incremental formulation.

The thicker green line in Figure 9 corresponds to the difference of the bent and the flat specimens, i.e. we assumed that residual stress far from the ends of

\footnotetext{
${ }^{14}$ Results of the fast algorithm (i.e. eq. 10 are not shown, but are practically the same.
} 
the extruded plate is substantially the same; finally the purple line corresponds to the line interpolating the difference data, which substantially corresponds to the expected results.

\section{Discussion}

This work discusses the implementation of the integral approach for residual stress measurement in the iDIC framework. Two main alternative algorithms are presented, the former based on a global minimization both in space and in time, the latter consisting in a cascade of minimizations, each identifying the parameters of the last drilling increment only, under the assumption that previously estimated stress values are correct.

The two algorithms give substantially the same results but the fast algorithm accesses image data and calibration coefficients sequentially, thus requiring significantly less memory. However its sequential approach makes it impossible to significantly improve it; in particular both SVD and Tikhonov regularization cannot be used.

Regarding the stabilization options discussed in section 3 , the SVD algorithm is effective when the fitting model contains terms unrelated to the real behavior but gives no advantage otherwise: thus, its use as a replacement of the LU solver in the "standard" formulation is mostly useless; moreover, replacing the normal equation approach with SVD results in a CPU and memory intensive algorithm with no improvement, as shown by numerical tests.

The oscillating behavior of results can be somewhat mitigated by using an incremental formulation, i.e. using the previous image instead of the first one as reference; as shown by Schajer and Rickert [33, the resulting solution matrix has a better condition number. However, incremental displacements are quite small, thus introducing some noise due to the relatively lower accuracy of DIC 
when compared to interferometric approaches.

In conclusion, Tikhonov regularization appears to be mandatory to obtain smooth results in real applications. It can be used either alone or in combination with all other algorithm variants (both SVD and the incremental approach) even though it is so effective that no extra "trick" is usually required. The Morozov criterion can be used to select the $\lambda$ parameter; alternatively, the solution time is so small that a simple trial and error search can also be easily performed.

\section{Acknowledgement}

The author wishes to thank Mr. Gianluca Marongiu and Mr. Daniele Lai for their support during data acquisition.

\section{References}

[1] ASTM E837-13a. Standard Test Method for Determining Residual Stresses by the Hole-Drilling Strain-Gage Method. American Society for Testing and Materials International, West Conshohocken, PA, 2013. doi:10.1520/E0837.

[2] D. V. Nelson and J. T. McCrickerd. Residual-stress determination through combined use of holographic interferometry and blind hole drilling. Experimental Mechanics, 26:371-378, 1986. ISSN 0014-4851.

[3] D. V. Nelson, A. Makino, and E. A. Fuchs. The holographic-hole drilling method for residual stress determination. Optics and Lasers in Engineering, 27:3-23, 1997.

[4] S. T. Lin, C.-T. Hsieh, and C. K. Lee. Full field phase-shifting holographic blind-hole techniques for in-plane residual stress detection. In Toshio Honda, editor, Int. Conf. on Applications of Optical Holography, 
volume 2577 of Proceedings of SPIE, pages 226-237, Bellingham, Washington, 1995.

[5] G. Nicoletto. Moiré interferometry determination of residual stresses in presence of gradients. Experimental Mechanics, 31(3):252-256, 1991. ISSN 0014-4851.

[6] Zhu Wu, Jian Lu, and Bongtae Han. Study of residual stress distribution by a combined method of moiré interferometry and incremental hole drilling, part i: theory. Journal of applied Mechanics, 65(4):837-843, 1998.

[7] R. C. Schwarz, L. M. Kutt, and J. M. Papazian. Measurement of residual stress using interferometric moiré: a new insight. Experimental Mechanics, 40(3):271-281, 2000. ISSN 0014-4851.

[8] M. Steinzig and E. Ponslet. Residual stress measurement using the hole drilling method and laser speckle interferometry: part i. Experimental Techniques, 27(3):43-46, May/June 2003. ISSN 1747-1567. doi: 10.1111/j. 1747-1567.2003.tb00114.x.

[9] E. Ponslet and M. Steinzig. Residual stress measurement using the hole drilling method and laser speckle interferometry part ii: analysis technique. Experimental Techniques, 27(4):17-21, July/August 2003. ISSN 1747-1567. doi: 10.1111/j.1747-1567.2003.tb00117.x.

[10] E. Ponslet and M. Steinzig. Residual stress measurement using the hole drilling method and laser speckle interferometry part iii: analysis technique. Experimental Techniques, 27(5):45-48, September/October 2003. ISSN 1747-1567. doi: 10.1111/j.1747-1567.2003.tb00130.x.

[11] Antonio Baldi. A new analytical approach for hole drilling residual stress 
analysis by full field method. Journal of Engineering Materials and Technology, 127(2):165-169, 2005. ISSN 0094-4289. doi: 10.1115/1.1839211.

[12] G. S. Schajer and M. Steinzig. Full-field calculation of hole drilling residual stresses from electronic speckle pattern interferometry data. Experimental Mechanics, 45(6):526-532, december 2005. ISSN 0014-4851.

[13] G.S. Schajer. Advances in hole-drilling residual stress measurements. Experimental Mechanics, 50(2):159-168, 2010. ISSN 0014-4851.

[14] G.S. Schajer, B. Winiarski, and P. J. Withers. Hole-drilling residual stress measurement with artifact correction using full-field dic. Experimental Mechanics, 53(2):255-265, 2013. ISSN 0014-4851.

[15] Julien Réthoré, Stéphane Roux, and François Hild. An extended and integrated digital image correlation technique applied to the analysis of fractured samples: The equilibrium gap method as a mechanical filter. European Journal of Computational Mechanics/Revue Européenne de Mécanique Numérique, 18(3-4):285-306, 2009.

[16] Gilles Besnard, François Hild, and Stéphane Roux. "finite-element" displacement fields analysis from digital images: application to portevin-le châtelier bands. Experimental Mechanics, 46(6):789-803, 2006.

[17] Antonio Baldi. Residual stress measurement using hole drilling and integrated digital image correlation techniques. Experimental Mechanics, 54 (3):379-391, 2013. ISSN 0014-4851. doi: 10.1007/s11340-013-9814-6.

[18] Antonio Baldi. Residual stress analysis of orthotropic materials using integrated digital image correlation. Experimental Mechanics, 54(7):1279-1292, 2014. ISSN 0014-4851. doi: 10.1007/s11340-014-9859-1. 
[19] Antonio Baldi. Sensitivity analysis of i-DIC approach for residual stress measurement in orthotropic materials. In Sven Bossuyt, Gary Schajer, and Alberto Carpinteri, editors, Residual Stress, Thermomechanics 8 Infrared Imaging, Hybrid Techniques and Inverse Problems, volume 9, pages 355-362. Springer, 2016. ISBN 978-3-319-21764-2. doi: 10.1007/978-3-319-21765-9.

[20] Michael A. Sutton, Jean-José Orteu, and Hubert Schreier. Image Correlation for Shape, Motion and Deformation Measurements: Basic Concepts, Theory and Applications. Springer, NY, USA, 2009. ISBN 978-0-38778746-6. doi: 10.1007/978-0-387-78747-3.

[21] B. Pan, H. Xie, and Z. Wang. Equivalence of digital image correlation criteria for pattern matching. Applied Optics, 49(28):5501-5509, 2010.

[22] Bruce D Lucas and Takeo Kanade. An iterative image registration technique with an application to stereo vision. In Proceedings of Imaging Understanding Workshop, volume 130, pages 121-130, 1981.

[23] Jérémie Rupil, Stéphane Roux, François Hild, and Ludovic Vincent. Fatigue microcrack detection with digital image correlation. The Journal of Strain Analysis for Engineering Design, 46(6):492-509, 2011.

[24] Benoît Blaysat, Johan PM Hoefnagels, Gilles Lubineau, Marco Alfano, and Marc GD Geers. Interface debonding characterization by image correlation integrated with double cantilever beam kinematics. International Journal of Solids and Structures, 55:79-91, 2015.

[25] G. S. Schajer. Measurement of non-uniform residual stresses using the hole-drilling method. part i - stress calculation procedures. Journal of Engineering Materials and Technology, 110(4):338-343, 1988. 
[26] J Neggers, Johan PM Hoefnagels, MGD Geers, François Hild, and Stéphane Roux. Time-resolved integrated digital image correlation. International Journal for Numerical Methods in Engineering, 103(3):157-182, 2015.

[27] Gilles Besnard, Hugo Leclerc, François Hild, Stéphane Roux, and Nicolas Swiergiel. Analysis of image series through global digital image correlation. The Journal of Strain Analysis for Engineering Design, 47(4): $214-228,2012$.

[28] W. H. Press, S. A. Teukolsky, W. T. Vetterling, and B. P. Flannery. Numerical Recipes. The Art of Scientific Computing. Cambridge University Press, 3rd edition, 2007.

[29] Gene H. Golub and Charles F. Van Loan. Matrix computations. John Hopkins University Press, Balrimore, Maryland, 4 edition, 2013. ISBN 978-1-4214-0794-4.

[30] Andreu Nikolaevich Tikhonov, A. Goncharsky, V.V. Stepanov, and Anatoly G. Yagola. Numerical methods for the solution of ill-posed problems, volume 328 of Mathematics and Its Applications. Springer, Netherlands, 1995. ISBN 978-0-7923-3583-2. doi: 10.1007/978-94-015-8480-7. Originally published in Russian.

[31] G. S. Schajer and M. B. Prime. Use of inverse solutions for residual stress measurements. Journal of Engineering Materials and Technology, 128:375382, 2006. doi:dx.doi.org/10.1115/1.2204952.

[32] Gary S Schajer. Hole-drilling residual stress profiling with automated smoothing. Journal of engineering materials and technology, 129(3):440445, 2007. doi: 10.1115/1.2744416.

[33] Gary S Schajer and Theo J Rickert. Incremental computation technique 
for residual stress calculations using the integral method. Experimental Mechanics, 51(7):1217-1222, 2011. doi: 10.1007/s11340-010-9408-5.

[34] Jean-José Orteu, Dorian Garcia, Laurent Robert, and Florian Bugarin. A speckle texture image generator. In Speckle06: speckles, from grains to flowers, volume 6341, page $63410 \mathrm{H}$. International Society for Optics and Photonics, 2006.

[35] Antonio Baldi and Filippo Bertolino. Experimental analysis of the errors due to polynomial interpolation in digital image correlation. Strain, 51(3): 248-263, 2015. ISSN 1475-1305. doi: 10.1111/str.12137.

[36] Antonio Baldi and Filippo Bertolino. Assessment of h-refinement procedure for global digital image correlation. Meccanica, 51(4):979-991, 2016.

[37] Antonio Baldi. Digital image correlation and color cameras. Experimental Mechanics, 58(2):315-333, 2018. ISSN 0014-4851. doi: 10.1007/ s11340-017-0347-2.

\section{A Notes on Numerical Implementation}

The described algorithms require iterating the solution procedure, thus, an efficient implementation should cache the calibration coefficients. However, this has to be done with care: $P^{u}, Q^{u}, \ldots, T^{v}$ depend on $\theta$ and on the $A, B, \ldots$, $G$ coefficients. These in turn depend on material properties and on the (normalized) radius. Thus, it is possible either to cache the $A, \ldots, G$ coefficients (the intermediate values) or the final set $\left(P^{u}, \ldots, T^{v}\right)$. The former solution corresponds to a minimal level of caching because the $A, \ldots, G$ coefficients depend on material and normalized radius only, thus, they can be easily stored either as a one-dimensional function of the radius (an interpolating function) or 
as a (small) list of values sampled at increasing radii. Using this solution, the $P_{i, j}^{u}, \ldots, T_{i, j}^{v}$ coefficients have to be (re)computed every time they are required, with a large computational overhead because each pixel $k$ of the image has a different polar coordinate with respect to the center of the hol $e^{15}$

Instead, if the computed $P_{i, j}^{u}, \ldots, T_{i, j}^{v}$, are stored in matrices, the above described procedure has to be performed only once, with obvious improvements in terms of $\mathrm{CPU}$ usage. However, this approach (full caching) requires a significant memory storage: indeed, assuming to perform $m$ drill increments, the number of matrices is

$$
6(1+2+3+\cdots+m)=6 \frac{m(m+1)}{2}=3 m(m+1)
$$

where the six appearing in front of the summation accounts for the six terms $\left(P^{u}, Q^{u}, \ldots, T^{v}\right)$ involved in (3).

To give some numbers, let assume that a 1 Mpixel camera is used to image 15 drilling increments; using these parameters, each of the $P^{u}, \ldots, T^{v}$ matrices requires $4 \mathrm{MiB}$ (we are assuming that 32 bit floating point numbers are used to store real values). Thus, to store all calibration coefficients in memory, you need $3 \times 15 \times(15+1) \times 4=2880 \mathrm{MiB}$ of storag $\AA^{16}$ Our empirical tests show that full caching is not required because solving the problems shown in the article requires a few seconds using an i7@3.4GHz processor and a minimal level of caching.

\footnotetext{
${ }^{15}$ Looking at 4 it is apparent that the computation of each set of coefficients related to given $i$ and $j$ values requires at least $6 \cdot n$ function evaluations and $4 \cdot n$ trigonometric computations. However, as $\theta$ depends on pixel coordinates only, both $\sin (\theta), \cos (\theta), \sin (2 \theta)$ and $\cos (2 \theta)$ can be pre-computed and stored in four matrices.

${ }^{16}$ This estimation moves to $7800 \mathrm{MiB}$ using 25 drill increments and to $15120 \mathrm{MiB}$ for 35 .
} 